\title{
Globular clusters and the evolution of their multiple stellar populations
}

\author{
W. Chantereau ${ }^{1}$, C. Charbonnel ${ }^{1,2}$ and G. Meynet ${ }^{1}$ \\ ${ }^{1}$ Department of Astronomy, University of Geneva, \\ Chemin des Maillettes 51, CH-1290 Versoix, Switzerland \\ email: william.chantereau@unige.ch \\ ${ }^{2}$ RAP, UMR 5277 CNRS and Université de Toulouse, \\ 14 Av. E. Belin, F-31400 Toulouse, France
}

\begin{abstract}
Our knowledge of the formation and early evolution of globular clusters (GCs) has been totally shaken with the discovery of the peculiar chemical properties of their long-lived host stars. Therefore, the interpretation of the observed Colour Magnitude Diagrams (CMD) and of the properties of the GC stellar populations requires the use of new stellar models computed with relevant chemical compositions. In this paper we use the grid of evolution models for low-mass stars computed by Chantereau et al. (2015) with the initial compositions of secondgeneration stars as predicted by the fast rotating massive stars scenario to build synthesis models of GCs. We discuss the implications of the assumed initial chemical distribution on $13 \mathrm{Gyr}$ isochrones. We build population synthesis models to predict the fraction of stars born with various helium abundances in present day globular clusters (assuming an age of 13 Gyr). With the current assumptions, $61 \%$ of stars on the main sequence are predicted to be born with a helium abundance in mass fraction, $\mathrm{Y}_{\text {ini }}$, smaller than 0.3 and only $11 \%$ have a $\mathrm{Y}_{\text {ini }}$ larger than 0.4. Along the horizontal branch, the fraction of stars with $Y_{\text {ini }}$ inferior to 0.3 is similar to that obtained along the main sequence band $(63 \%)$, while the fraction of very He-enriched stars is significantly decreased (only $3 \%$ with $\mathrm{Y}_{\text {ini }}$ larger than 0.38 ).
\end{abstract}

Keywords. globular clusters: general, Hertzsprung-Russell diagram, stars: evolution, stars: lowmass, stars: abundances

\section{Introduction}

It is now well accepted that GCs host at least two populations of low-mass stars, one presenting the chemical patterns typical of the protoglobular cluster gas and of halo stars of similar metallicity (first population, 1P), while the other one displays peculiar abundances (second population, 2P). This overall picture has been set up thanks to numerous spectroscopic surveys (see e.g. Gratton et al. 2001; Carretta et al. 2009a, 2009b), and the famous O-Na anti-correlation has been adopted as the main characteristic of a bona-fide GC (Carretta et al. 2010). In addition, with the advent of high resolution photometry, this paradigm has been strongly supported by the discovery of multiple discrete structures in CMDs of individual GCs (see e.g. Bedin et al. 2004; Piotto et al. 2007; Piotto 2009; Milone et al. 2013). Different scenarios implying different polluters have been developed to explain the nucleosynthetic sources of ashes of H-burning at high temperature needed to explain the chemical composition of $2 \mathrm{P}$ stars. The main polluters advocated in the literature are fast rotating massive stars (FRMS, Decressin et al. 2007; Krause et al. 2013), asymptotic giant branch stars (AGB, Ventura et al. 2001, 2013), massive binary stars (de Mink et al. 2009), supermassive stars (Denissenkov \& Hartwick 2014), and also low-mass pre-main sequence stars coupled with interactive binaries/and or rotating massive stars (Bastian et al. 2013). 


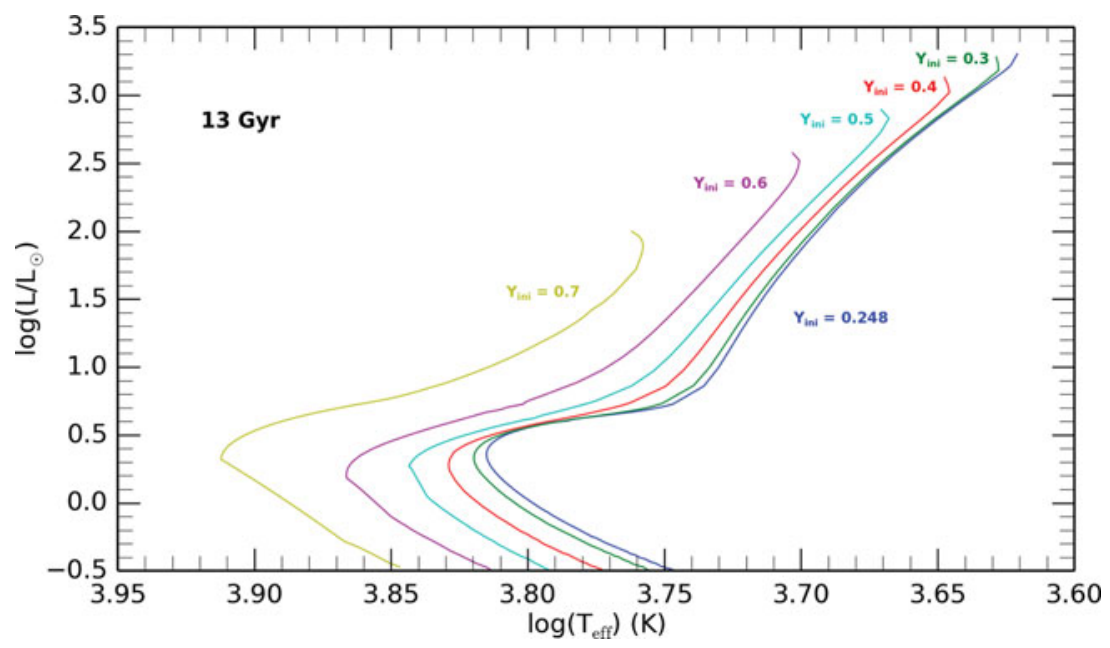

Figure 1. Theoretical isochrones at 13 Gyr for different initial helium composition between 0.248 and 0.7 (mass fraction).

Despite the numerous differences between the scenarios developed to explain the typical chemical patterns of GCs, it is widely accepted by the community that $2 \mathrm{P}$ stars have formed with a higher helium content than their $1 \mathrm{P}$ counterparts. It is then mandatory to take into account the impact of various helium content on the evolution of stars in order to provide a fully consistent scenario. Each of the above scenarios predict different initial helium content in the $2 \mathrm{P}$ stars. The various initial He distributions associated will then evolve into different distributions as a result of the evolution of stars and give therefore various outcomes for the helium distribution in the present day GCs. This is the point that we study in the present paper. More specifically, we start from the initial abundances predicted by the FRMS scenario and we predict the helium distribution that one should observe in the cluster NGC 6752. Although helium abundances remain very difficult to measure directly by spectroscopy in cool stars, there exist indirect ways to constrain the present day helium distribution in GCs (see e.g. Salaris et al. 2004).

\section{General framework}

In the FRMS scenario, $2 \mathrm{P}$ stars form in the immediate vicinity of very massive and fast rotating stars (into the mass range $25-120 \mathrm{M}_{\odot}$ as investigated by Decressin et al. 2007a,b; Krause et al. 2013) from their H-burning ashes diluted at various degrees with the surrounding intra-cluster pristine gas. Therefore $2 \mathrm{P}$ stars are mainly $\mathrm{C}-, \mathrm{O}-$, and $\mathrm{Mg}$ depleted while they are $\mathrm{He}-, \mathrm{N}-, \mathrm{Na}-$, and Al-enriched, compared to their $1 \mathrm{P}$ counterparts that display a chemical composition typical of the pristine intra-cluster material and of the surrounding halo field stars. At the metallicity investigated in this study $([\mathrm{Fe} / \mathrm{H}]=$ -1.75), $2 \mathrm{P}$ stars are expected to be born with $\mathrm{Y}_{\text {ini }}$ ranging between 0.248 (as 1P) and 0.8 .

\section{Isochrones}

Stellar models enriched in helium have a higher luminosity and effective temperature ( $\left.\mathrm{T}_{\text {eff }}\right)$, thus evolve faster (see Chantereau et al. (2015), hereafter paper I, for more details). At a given age, He-enriched isochrones are thus located in the HRD at a higher $\mathrm{T}_{\text {eff }}$, and are populated by lower masses than the reference isochrone $\left(Y_{\mathrm{ini}}=0.248\right.$, see fig. 1$)$. 
More quantitatively, the effective temperature of stars with a solar luminosity are hotter by $1,400 \mathrm{~K}$ when their initial helium abundance is 0.7 instead of 0.248 . At this luminosity, the $2 \mathrm{P}$ stars with $\mathrm{Y}_{\text {ini }}=0.7$ display a mass lower by $\sim 0.4 \mathrm{M}_{\odot}$ than their $1 \mathrm{P}$ counterparts. These differences are much smaller for models moderately enriched in helium, still at solar luminosity, we expect 'only' an increase of less than $\sim 100 \mathrm{~K}$ between the reference isochrone and the $\mathrm{Y}_{\mathrm{ini}}=0.3$ one, which is similar to the error in $\mathrm{T}_{\text {eff }}$. Along the RGB, we expect the same behaviour for the He-rich models as for the previous phase, i.e. mainly an increase of the $\mathrm{T}_{\text {eff }}$ with the helium enhancement. This time the dispersion is smaller than the one at solar luminosity, slightly above $700 \mathrm{~K}$ for the $\mathrm{Y}_{\text {ini }}=0.7$ case in the middle of the RGB $\left(\log \left(\mathrm{L} / \mathrm{L}_{\odot}\right)=1.5\right)$. Again, for lower helium enrichments (e.g. $\mathrm{Y}_{\text {ini }}=0.4$ ), the increase in $\mathrm{T}_{\text {eff }}$ is only $\sim 100 \mathrm{~K}$. Finally, as expected (see paper $\mathrm{I}$ and Chantereau et al., in prep.), the RGB-tip is fainter for He-enriched models, therefore the maximum helium enrichment and the temperature spread decreases as we go to higher luminosities.

\section{Synthetic globular clusters}

\subsection{Initial conditions and basic assumptions}

The construction of a synthetic GC is based on the grid of models (see paper I) assuming a initial mass function (IMF) and an initial distribution for the initial helium mass fraction of the stars populating the cluster. We use a Paresce \& De Marchi (2000) IMF for masses below $0.85 \mathrm{M}_{\odot}$, and a Salpeter IMF (Salpeter 1955) with $\alpha=2.35$ for higher masses. The distribution of the initial helium distribution is build to retrieve the sodium distribution of the bright RGB stars of NGC 6752 (Carretta, 2013), a GC which metallicity is close to the one of our grid $([\mathrm{Fe} / \mathrm{H}]=-1.56$, Carretta et al. $2009 \mathrm{a})$. Indeed from the sodium abundance we can infer the helium content thanks to the helium-sodium correlation provided by the FRMS scenario (see fig. 1 in the paper I). Then with Monte-Carlo simulations of $300^{\prime} 000$ stars $\left(0.3-1.0 \mathrm{M}_{\odot}\right)$, by iteration on the $\mathrm{Y}_{\text {ini }}$ distribution we reproduce this sodium distribution observed on the RGB at 13 Gyr (13.4 \pm 1.1 Gyr for NGC 6752, Gratton et al. 2003).

The initial helium mass fraction distribution inferred this way (see fig. 2) is composed of $49 \%$ of stars displaying an initial helium enrichment above 0.3 and $21 \%$ above $0.4 \dagger$. After 13 Gyr, from the initial sample of 300'000 stars, only 200'000 stars $(\mathrm{M}=0.30$ $\left.0.81 \mathrm{M}_{\odot}\right)$ are still alive.

\subsection{Helium distribution along the HRD at 13 Gyr}

In this section we present the distribution of stars according to their helium content at birth $\left(\mathrm{Y}_{\mathrm{ini}}\right)$ and after $13 \mathrm{Gyr}$ of evolution. This is shown in fig. 3 where we present the relative number of stars as a function of their initial helium content, for different regions of the HRD.

Along the MS (here $-0.5 \leqslant \log \left(\frac{L}{L_{\odot}}\right) \leqslant 0.3$; top panel in fig. 3), the highest initial helium content that we obtain is 0.724 , which implies in principle a broadening of the MS band in the HRD by about 1,900 K. However in this domain in luminosity, only $28 \%$ of the main sequence stars were born with $\mathrm{Y}_{\text {ini }}$ between 0.3 and $0.4,11 \%$ with $\mathrm{Y}_{\text {ini }}$ higher than 0.4 , and $2 \%$ with $\mathrm{Y}_{\text {ini }}$ higher than 0.6 . Therefore, the photometric impact of the

$\dagger$ We used a grid of standard stellar models for low-mass stars $\left(0.3-1.0 \mathrm{M}_{\odot}\right)$ at low metallicity $([\mathrm{Fe} / \mathrm{H}]=-1.75)$, with an alpha-enhancement of +0.3 and initial mass fraction of helium between 0.248 and 0.8 (paper I) 


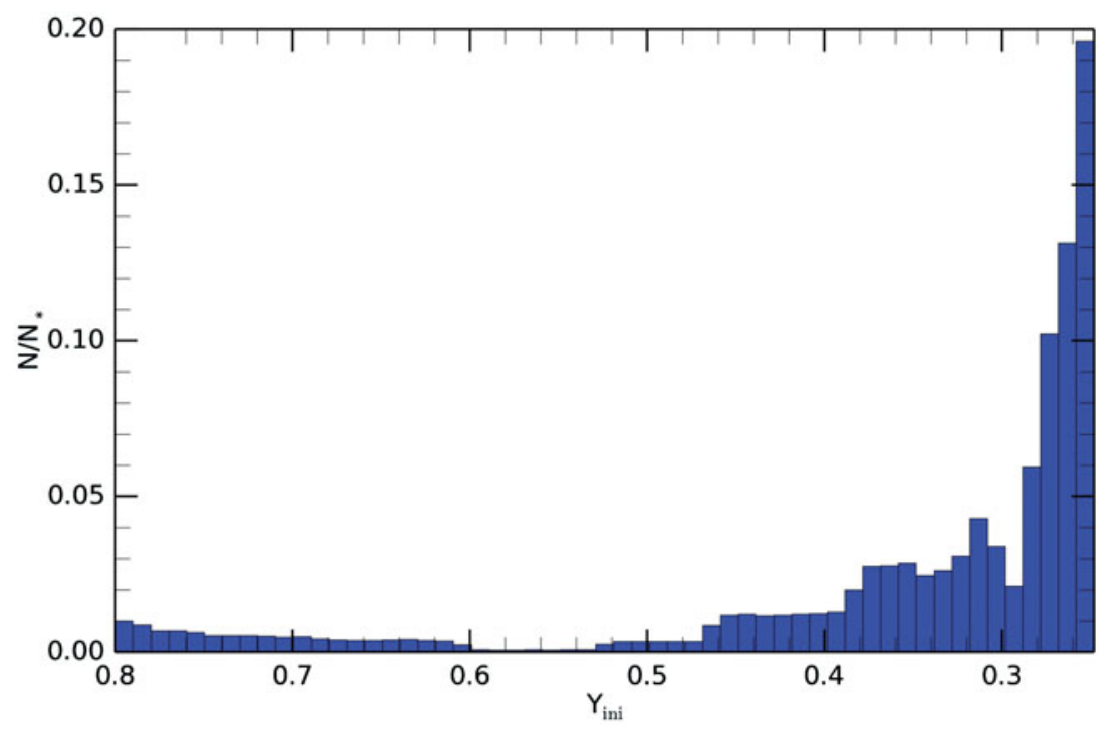

Figure 2. Initial distribution of the helium mass fraction for our sample of 300'000 stars with assumptions described in $\S 4.1$.

high helium tail $\left(\mathrm{Y}_{\text {ini }}>0.4\right)$ would be extremely moderate.

On the bright part of the RGB $\left(\log \left(\frac{L}{L_{\odot}}\right) \geqslant 1.2\right.$; third panel in fig. 3, upper RGB), the maximum $\mathrm{Y}_{\mathrm{ini}}$ found is nearly the same as on the MS (0.729), and the proportion of stars born with a $\mathrm{Y}_{\text {ini }}$ above 0.3 is $38 \%$, similar to the one found for the MS. In this luminosity domain, only $10 \%$ of the stars were born with an initial helium content above 0.4 , and $2 \%$ with $\mathrm{Y}_{\text {ini }}$ higher than 0.6.

The RGB-tip being fainter for stars with higher initial helium content (see fig 1), as we go to higher luminosities, the maximum $Y_{\text {ini }}$ and the fraction of present He-rich stars drops quickly, therefore the distribution of helium on the RGB is very sensitive to the bin of luminosity chosen.

At 13 Gyr all the stars with an initial helium content above 0.41 evolve directly towards the cooling white dwarf curve without undergoing the He-flash and end their life as He-WD. Therefore we do not expect to see stars born with a higher helium content on the horizontal branch (HB) and a fortiori on the AGB (see Charbonnel et al. 2013). A star with a $\mathrm{Y}_{\text {ini }}=0.41$ have also the lower mass on this phase $\left(0.473 \mathrm{M}_{\odot}\right)$ while the $1 \mathrm{P}$ stars on this phase have a current mass of $0.6 \mathrm{M}_{\odot}$. We also expect to still find $37 \%$ of stars born with a helium content above $0.3 \mathrm{Y}_{\text {ini }}$ but only $3 \%$ with a helium value between 0.38 and 0.41 .

Surprisingly, we find that the maximum $\mathrm{Y}_{\mathrm{ini}}$ for stars on the HB is similar to the maximum He enrichment for $2 \mathrm{P}$ stars in the AGB scenario ( 0.38, e.g. Doherty et al. 2014$)$. Therefore we see that the determination of He for HB stars can not help discriminating between the two scenarios.

Concerning the AGB phase, the maximum $Y_{\text {ini }}$ value found is even lower due to stars that ignite helium later on the HB (hot and late-hot flashers, see e.g. Miller Bertolami et al. 2008), and that miss the AGB phase, the so-called AGB-manqué stars (see e.g. 


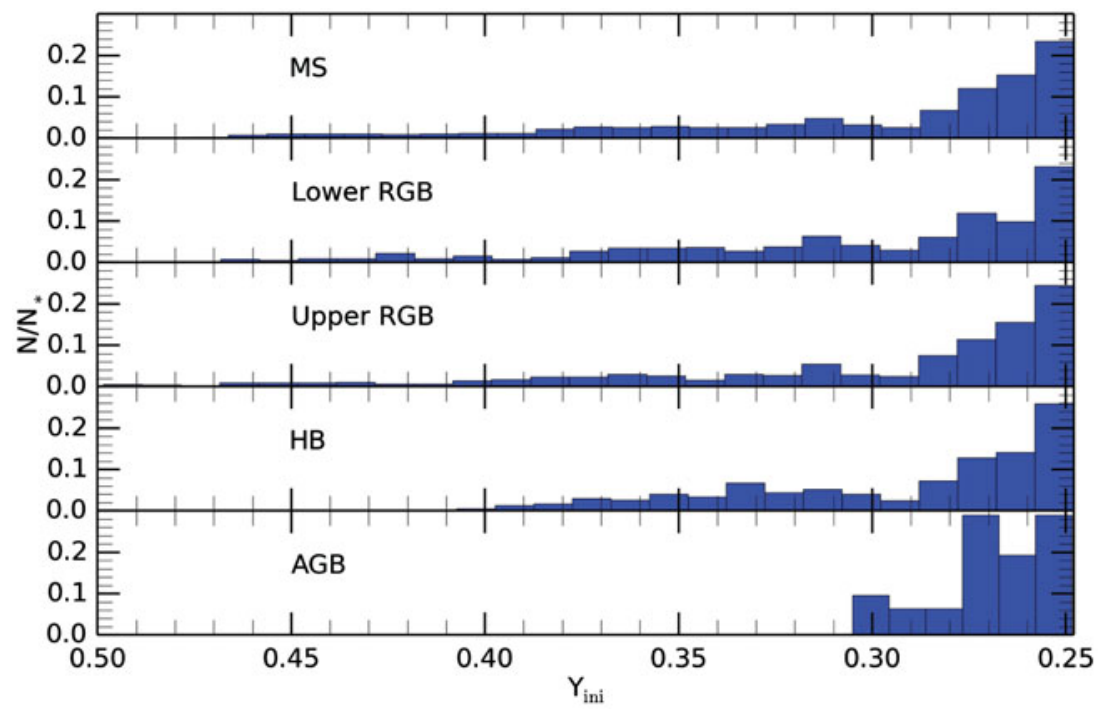

Figure 3. $\mathrm{Y}_{\mathrm{ini}}$ distribution along a GC at 13 Gyr. The MS corresponds to a bin of luminosity between $\log \left(\frac{L}{L_{\odot}}\right)=-0.5$ and 0.3 , The RGB phase is separated in two parts, the lower one displays the distribution for a bin of luminosity between $\log \left(\frac{L}{L_{\odot}}\right)=0.8$ and 1.2 while the upper part shows all the brighter stars.

Charbonnel et al. 2013, Cassisi et al. 2014). At 13 Gyr, the most helium enriched model displays an initial helium content of 0.305 and a mass of $0.536 \mathrm{M}_{\odot}$ whereas its $1 \mathrm{P}$ counterpart has a mass of $0.598 \mathrm{M}_{\odot}$. The maximal $\mathrm{Y}_{\text {ini }}$ value is very sensitive to the initial helium mass fraction distribution coupled to the IMF because of the very low number of stars at this phase (31 out of the 200 '000 here).

\section{Conclusion}

Starting with initial helium abundances for $2 \mathrm{P}$ stars as given by the FRMS models and using standard assumptions for the IMF, we present predictions for the helium distribution in a typical 13 Gyr old GC with $[\mathrm{Fe} / \mathrm{H}]=-1.75$.

At birth, $49 \%$ of the stars have a helium content above 0.3 , among which $21 \%$ have $\mathrm{Y}_{\text {ini }}$ higher than 0.4. At $13 \mathrm{Gyr}$, due to stellar evolution, the stars born with $\mathrm{Y}_{\text {ini }} \geqslant 0.3$ correspond to $39 \%$ of the stars present in the GC (sum over all the phases) among which only $10 \%$ born with a $\mathrm{Y}_{\text {ini }} \geqslant 0.4$.

The low fraction of stars with very high helium enrichments (or their total absence) shows that it would be complicated (or impossible) to use the maximum helium enrichment as a strong discriminating parameter between the different scenarios trying to explain this multiple populations phenomenon (for this typical age and metallicity).

\section{Acknowledgements}

We warmly thank Eugenio Carretta for having kindly provided us his valuable data on NGC 6752. We acknowledge support from the Swiss National Science Foundation (FNS). We thank the International Space Science Institute (ISSI, Bern, CH) for welcoming the activities of ISSI Team 271 "Massive Star Clusters across the Hubble Time" (2013 - 2015; team leader C.C.). C.W. acknowledges financial support from the "Société Académique de Genève" and the "Fondation Ernst et Lucie Schmidheiny". 


\section{References}

Bastian, N., Lamers, H. J. G. L. M., de Mink, S. E., Longmore, S. N., Goodwin, S. P., \& Gieles, M. 2013, MNRAS, 436, 2398

Bedin, L. R., Piotto, G., Anderson, J., Cassisi, S., King, I. R, Momany, Y., \& Carraro, G. 2004, ApJ, 605, L125

Carretta, E., Bragaglia, A., Gratton, R. G., Lucatello, S., Catanzaro, G., Leone, F., Bellazzini, M., Claudi, R., D’Orazi, V., Momany, Y., et al. 2009a, A\&A, 505, 117

Carretta, E., Bragaglia, A., Gratton, R., D’Orazi, V., \& Lucatello, S. 2009b, A\&A, 508, 695C

Carretta, E., Bragaglia, A., Gratton, R. G., Recio-Blanco, A., Lucatello, S., D’Orazi, V., \& Cassisi, S. 2010, A\&̈A, 516, A55

Carretta, E. 2013, A\&AA, 557, A128

Cassisi, S., Salaris, M., Pietrinferni, A., Vink, J. S., \& Monelli, M. 2014, A\&A, 571, 81C

Chantereau, W., Charbonnel, C., \& Decressin, T. 2015, A\&3A, 578, A117

Charbonnel, C., Chantereau, W., Decressin, T., Meynet, G., \& Schaerer, D. 2013, A\& A, 557, L17

Decressin, T., Charbonnel, C., \& Meynet, G. 2007a, A\&A, 475, 859

Decressin, T., Meynet, G., Charbonnel, C., Prantzos, N., \& Ekström, S. 2007b, A\&A, 464, 1029

de Mink, S. E., Pols, O. R., Langer, N., \& Izzard, R. G. 2009, A\&A A, 507, L1

Denissenkov, P. A. \& Hartwick, F. D. A. 2014, MNRAS, 437, L21

Doherty, C. L., Gil-Pons, P., Lau, H. H. B., Lattanzio, J. C., Siess, L., \& Campbell, S. 2014, MNRAS, 441, 582

Gratton, R. G., Bonifacio, P., Bragaglia, A., Carretta, E., Castellani, V., Centurion, M., Chieffi, A., Claudi, R., Clementini, G., D’Antona, F., et al. 2001, A\&A, 369, 87

Gratton, R. G., Bragaglia, A., Carretta, E., Clementini, G., Desidera, S., Grundahl, F., \& Lucatello, S. 2003 A\&A, 408, 529

Krause, M., Charbonnel, C., Decressin, T., Meynet, G., \& Prantzos, N. 2013, A\&A, 552, A121

Miller Bertolami, M. M., Althaus, L. G., Unglaub, K., \& Weiss, A. 2008, A\&A 491, 253

Milone, A. P., Marino, A. F., Piotto, G., Bedin, L. R., Anderson, J., Aparicio, A., Bellini, A., Cassisi, S., D'Antona, F., Grundahl, F., Monelli, M., \& Yong, D. 2013, ApJ, 767, 120

Paresce, F. \& De Marchi, G. 2000, ApJ, 534, 870

Piotto, G., Bedin, L. R., Anderson, J., King, I. R., Cassisi, S., Milone, A. P., Villanova, S., Pietrinferni, A., \& Renzini, A. 2007, ApJ, 661, L53

Piotto, G. 2009, IAUS, 258, 233P

Salaris, M., Riello, M., Cassisi, S., \& Piotto, G. 2004, A\&SA, 420, 911S

Salpeter, E. E. 1955, ApJ, 121, 161

Ventura, P., D'Antona, F., Mazzitelli, I., \& Gratton, R. 2001, ApJ, 550, L65

Ventura, P., Di Criscienzo, M., Carini, R., \& D'Antona, F. 2013, MNRAS, 431, 3642 